Supporting Information

\title{
Asymmetric Total Synthesis of (+)-Winchinine B
}

Zaimin Liu, Xiaolin Ju, Shiqiang Ma, Chenglong Du, Weiwei Zhang, Huilin Li, Xiaolei Wang,

Xingang Xie and Xuegong She*

State Key Laboratory of Applied Organic Chemistry, College of Chemistry and Chemical

Engineering, Lanzhou University, Lanzhou 730000, China

*E-mail: shexg@1zu.edu.cn

\section{Table of Contents}

1. Data Comparison of the Natural and Synthetic Samples................S1-S3

2. Copies of the Related NMR Spectra.................................S4-S27

3. HPLC spectra of S-1.............................................S28-S29 
1. Comparison of the data of our synthetic samples with those reported by literature.

\section{Winchinine B:}

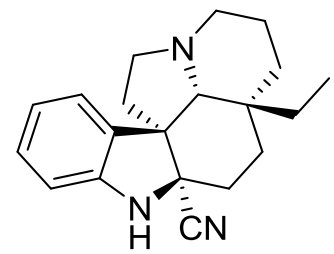

(+)-Winchinine B (1b)

${ }^{1} \mathrm{H}$ NMR, $400 \mathrm{M}^{\mathrm{NMR}}, \mathrm{CDCl}_{3}$

\begin{tabular}{|c|c|c|c|c|}
\hline $\begin{array}{l}\text { Natural sample } \\
\delta \text { (ppm) }\end{array}$ & $J(\mathrm{~Hz})$ & $\begin{array}{l}\text { Synthetic sample } \\
\delta \text { (ppm) }\end{array}$ & $J(\mathrm{~Hz})$ & $\begin{array}{l}\text { Err } \\
\text { (natural-synthetic) } \\
\Delta \delta(\mathrm{ppm})\end{array}$ \\
\hline $7.05(\mathrm{~m}, 2 \mathrm{H})$ & & $7.05(\mathrm{~m}, 2 \mathrm{H})$ & & 0 \\
\hline $6.79(\mathrm{dd}, 1 \mathrm{H})$ & 7.6, 7.6 & $6.80(\mathrm{dd}, 1 \mathrm{H})$ & $7.4,7.4$ & -0.01 \\
\hline $6.63(\mathrm{~d}, 1 \mathrm{H})$ & 7.6 & $6.63(\mathrm{~d}, 1 \mathrm{H})$ & 7.7 & 0 \\
\hline $3.93(\mathrm{~s}, 1 \mathrm{H})$ & & $3.93(\mathrm{~s}, 1 \mathrm{H})$ & & 0 \\
\hline $3.25(\mathrm{td}, 1 \mathrm{H})$ & $9.2,4.3$ & $3.26(\mathrm{td}, 1 \mathrm{H})$ & $9.0,4.3$ & -0.01 \\
\hline $3.03(\mathrm{br} \mathrm{d}, 1 \mathrm{H})$ & 9.3 & $3.04(\mathrm{br} \mathrm{d}, 1 \mathrm{H})$ & 10.6 & -0.01 \\
\hline $2.66(\mathrm{~m}, 1 \mathrm{H})$ & & $2.67(\mathrm{~m}, 1 \mathrm{H})$ & & -0.01 \\
\hline $2.35(\mathrm{~s}, 1 \mathrm{H})$ & & $2.36(\mathrm{~s}, 1 \mathrm{H})$ & & -0.01 \\
\hline $2.28(\mathrm{dd}, 1 \mathrm{H})$ & $11.0,6.0$ & $2.29(\mathrm{~m}, 1 \mathrm{H})$ & & -0.01 \\
\hline $2.24(\mathrm{~d}, 1 \mathrm{H})$ & 9.2 & $2.24(\mathrm{~m}, 1 \mathrm{H})$ & & 0 \\
\hline $1.97(\mathrm{~m}, 2 \mathrm{H})$ & & $1.97(\mathrm{~m}, 2 \mathrm{H})$ & & 0 \\
\hline $1.78(\mathrm{~m}, 2 \mathrm{H})$ & & $1.77(\mathrm{~m}, 2 \mathrm{H})$ & & 0.01 \\
\hline $1.62(\mathrm{~d}, 1 \mathrm{H})$ & 13.7 & $1.62(\mathrm{~d}, 1 \mathrm{H})$ & 13.6 & 0 \\
\hline $1.5(\mathrm{~m}, 2 \mathrm{H})$ & & $1.49(\mathrm{~m}, 2 \mathrm{H})$ & & 0.01 \\
\hline $1.38(\mathrm{dd}, 1 \mathrm{H})$ & $14.5,7.5$ & $1.39(\mathrm{dd}, 1 \mathrm{H})$ & $14.4,7.4$ & -0.01 \\
\hline $1.12(\mathrm{dd}, 1 \mathrm{H})$ & $13.7,3.7$ & $1.13(\mathrm{~m}, 1 \mathrm{H})$ & & -0.01 \\
\hline $1.07(\mathrm{dd}, 1 \mathrm{H})$ & $13.4,3.7$ & $1.08(\mathrm{~m}, 1 \mathrm{H})$ & & -0.01 \\
\hline $0.91(\mathrm{dd}, 1 \mathrm{H})$ & $14.5,7.5$ & $0.92(\mathrm{~m}, 1 \mathrm{H})$ & & -0.01 \\
\hline $0.63(\mathrm{t}, 3 \mathrm{H})$ & 7.5 & $0.64(\mathrm{t}, 3 \mathrm{H})$ & 7.5 & -0.01 \\
\hline
\end{tabular}




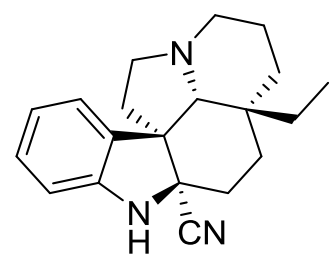

(+)-Winchinine B (1b)

${ }^{13} \mathrm{C}$ NMR, $100 \mathrm{M} \mathrm{NMR}, \mathrm{CDCl}_{3}$

\begin{tabular}{|c|c|c|}
\hline $\begin{array}{l}\text { Natural sample } \\
\delta(\mathrm{ppm})\end{array}$ & $\begin{array}{l}\text { Synthetic sample } \\
\delta(\mathrm{ppm})\end{array}$ & $\begin{array}{l}\text { Err } \\
\text { (natural-synthetic) } \\
\Delta \delta(\mathrm{ppm})\end{array}$ \\
\hline 146.5 & 146.5 & 0 \\
\hline 133.3 & 133.2 & 0.1 \\
\hline 128.2 & 128.2 & 0 \\
\hline 123.3 & 123.3 & 0 \\
\hline 122.2 & 122.2 & 0 \\
\hline 120.8 & 120.8 & 0 \\
\hline 111.1 & 111.0 & 0.1 \\
\hline 69.7 & 69.7 & 0 \\
\hline 64.8 & 64.8 & 0 \\
\hline 57.9 & 57.9 & 0 \\
\hline 53.5 & 53.4 & 0.1 \\
\hline 51.8 & 51.8 & 0 \\
\hline 36.7 & 36.7 & 0 \\
\hline 36.0 & 36.0 & 0 \\
\hline 34.3 & 34.3 & 0 \\
\hline 32.7 & 32.7 & 0 \\
\hline 29.9 & 29.9 & 0 \\
\hline 22.1 & 22.1 & 0 \\
\hline 21.6 & 21.6 & 0 \\
\hline 7.1 & 7.0 & 0.1 \\
\hline
\end{tabular}


Natural sample: IR (KBr) $v_{\max } 3323,2937,2790,2362,1680,1540,1508,1457,1383,1328$, $1179,1141,747 \mathrm{~cm}^{-1}$.

Synthetic sample: IR (neat) $v_{\max } 3332,2935,2788,2374,1687,1545,1510,1466,1377,1330$, $1181,1141,734 \mathrm{~cm}^{-1}$.

Natural sample: HRESIMS: calcd for $\mathrm{C}_{20} \mathrm{H}_{26} \mathrm{~N}_{3}[\mathrm{M}+\mathrm{H}]^{+}$308.2121, found 308.2128.

Synthetic sample: HRMS (ESIMS): calcd for $\mathrm{C}_{20} \mathrm{H}_{26} \mathrm{~N}_{3}[\mathrm{M}+\mathrm{H}]^{+} 308.2121$, found 308.2123.

Optical rotation: Natural sample: $[\alpha]_{\mathrm{D}}^{25}+19.2\left(\mathrm{CH}_{3} \mathrm{OH}, \mathrm{c}=1.0\right)$.

Synthetic sample: $[\alpha]_{\mathrm{D}}^{24.5}+35.0\left(\mathrm{CH}_{3} \mathrm{OH}, \mathrm{c}=1.0\right)$ 

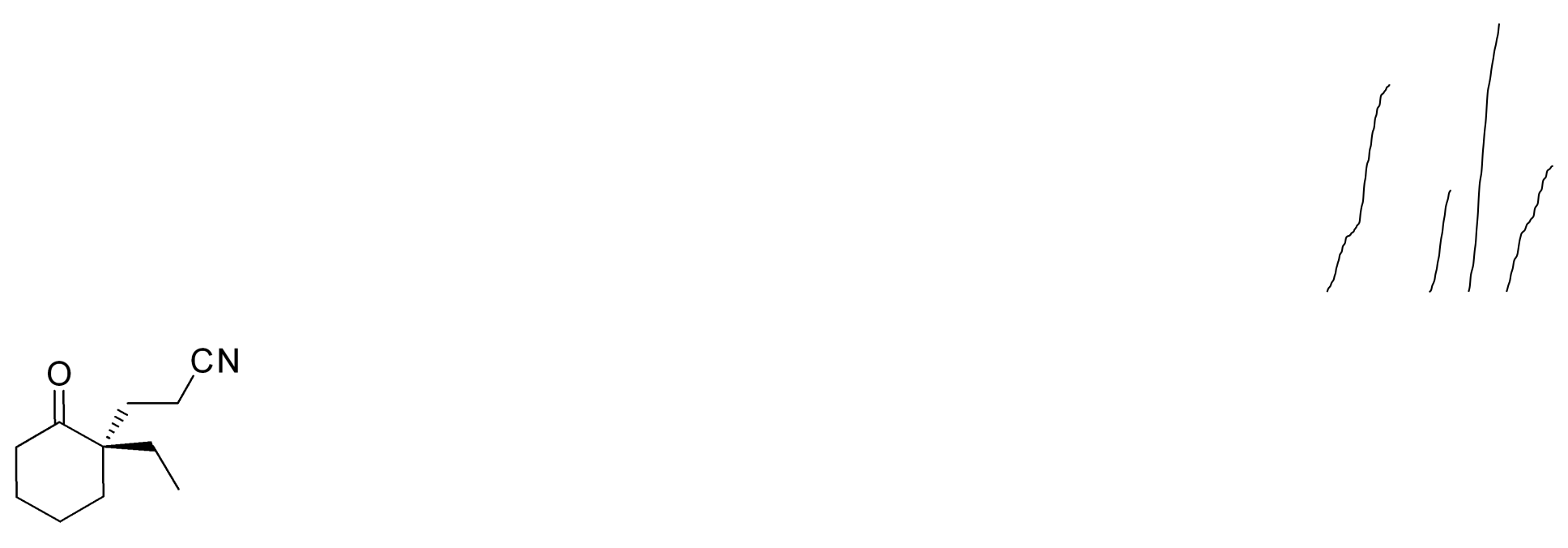

10

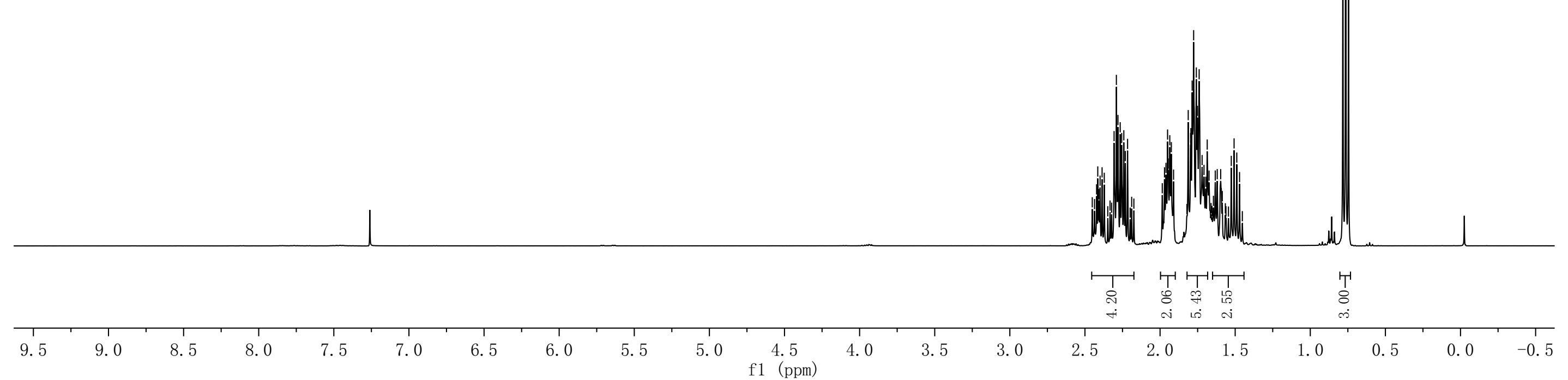



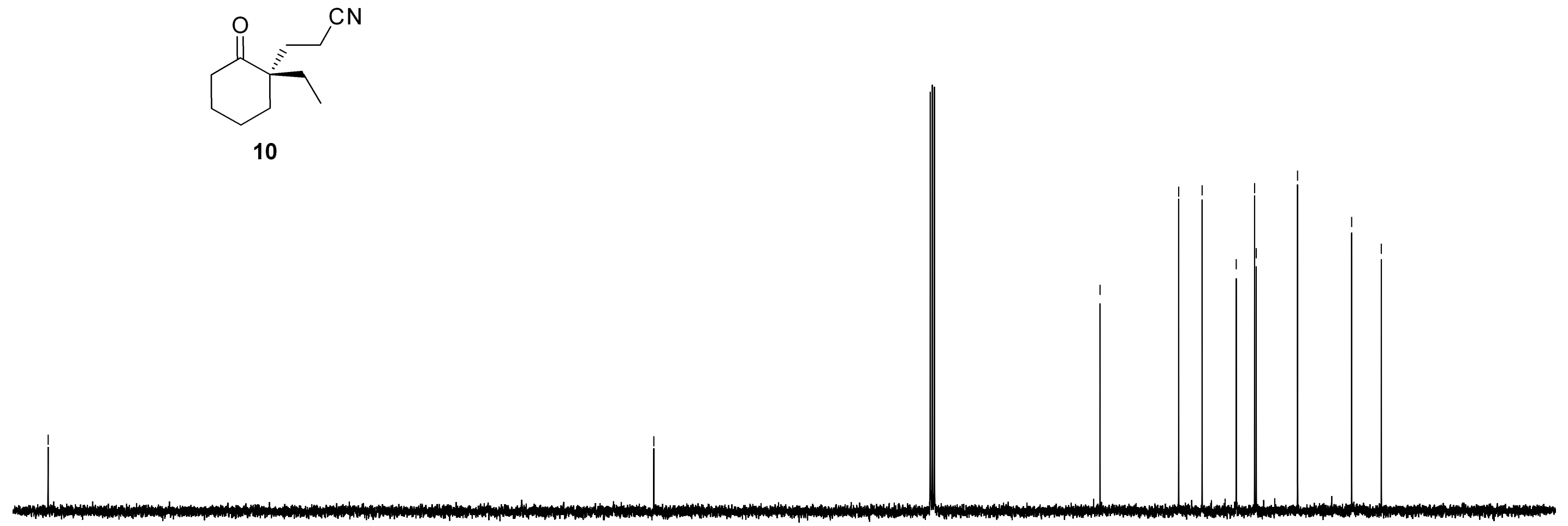

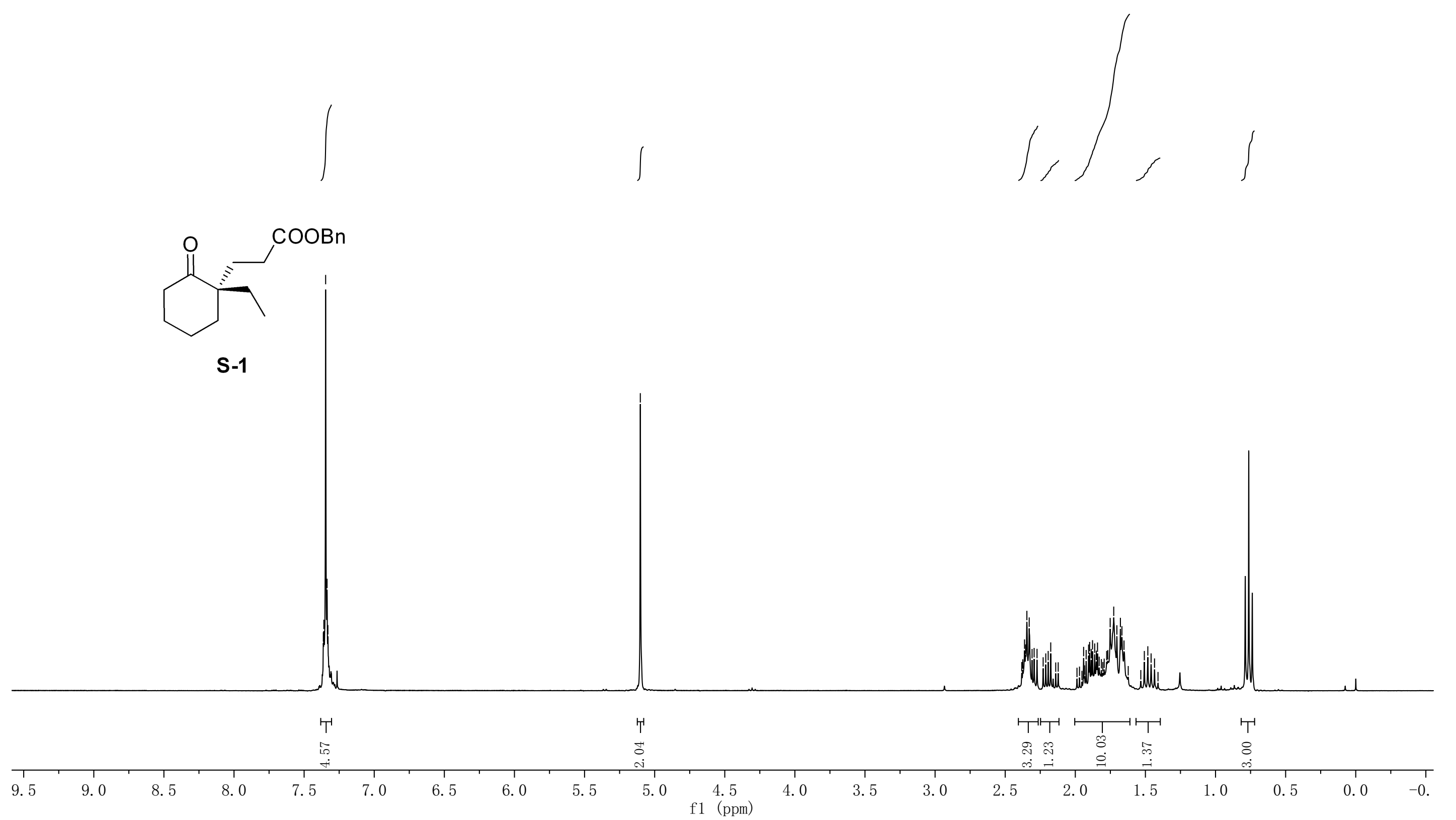


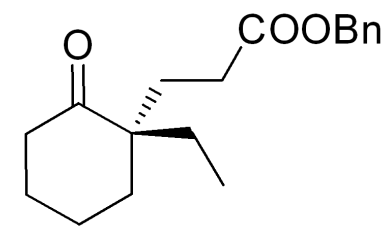

S-1

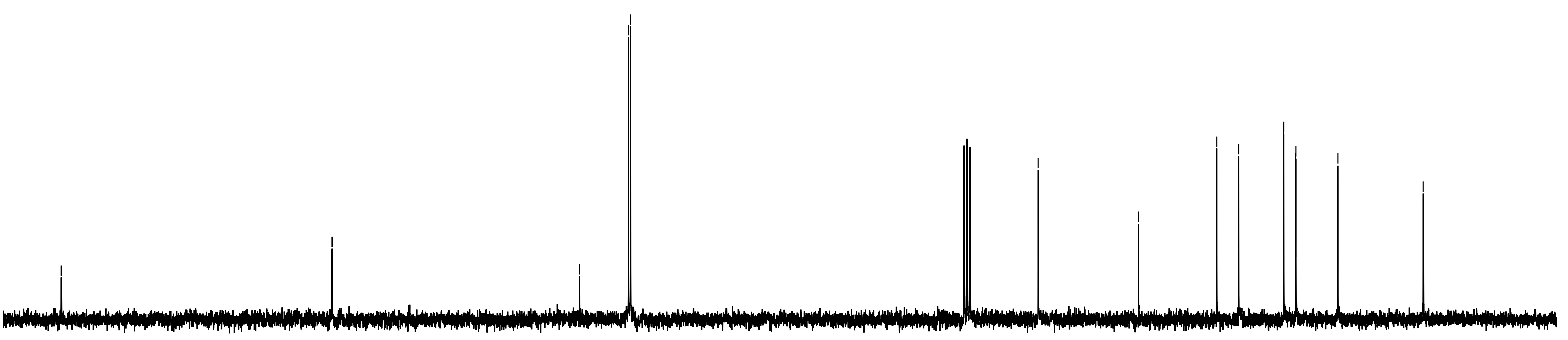



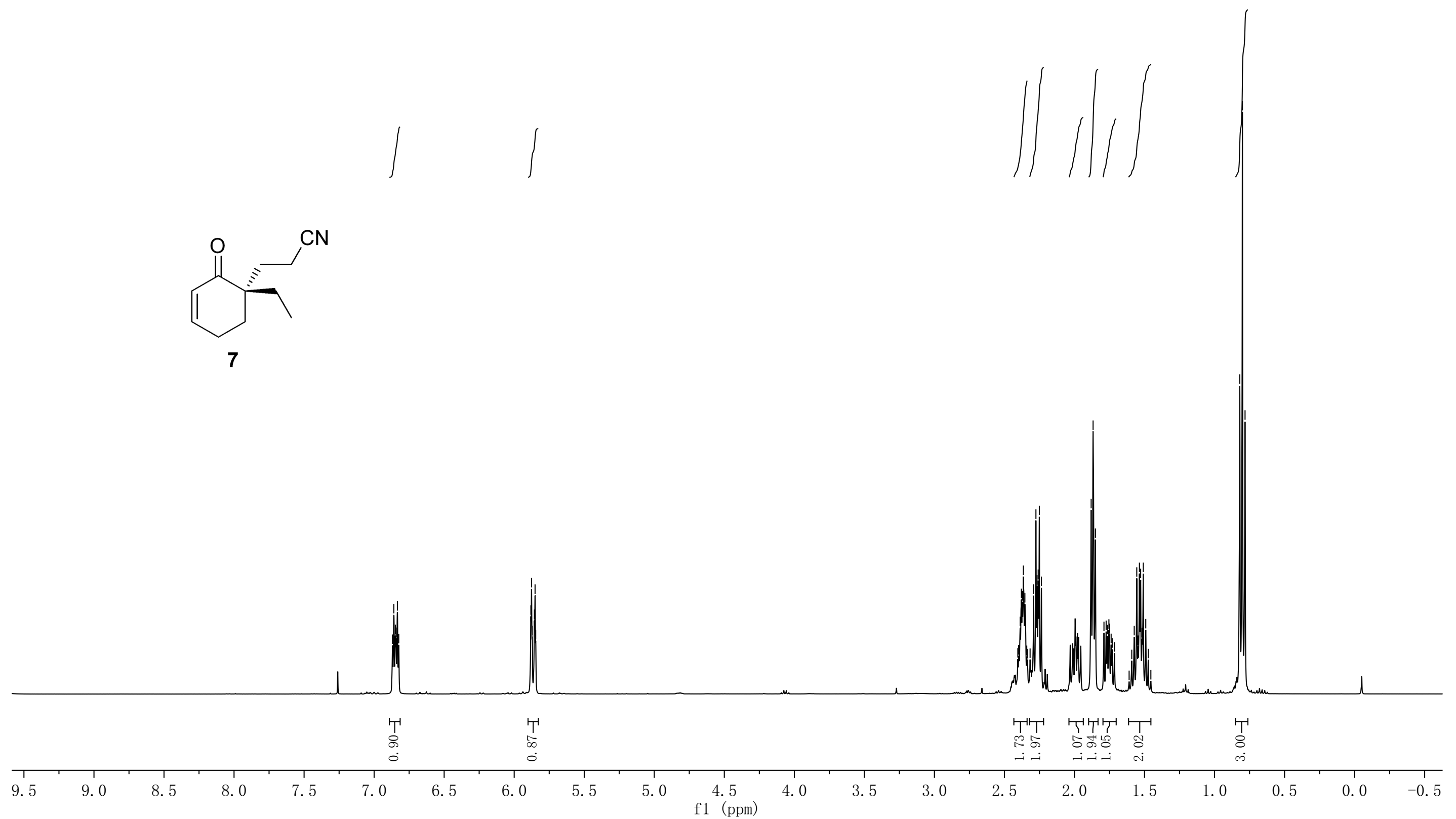

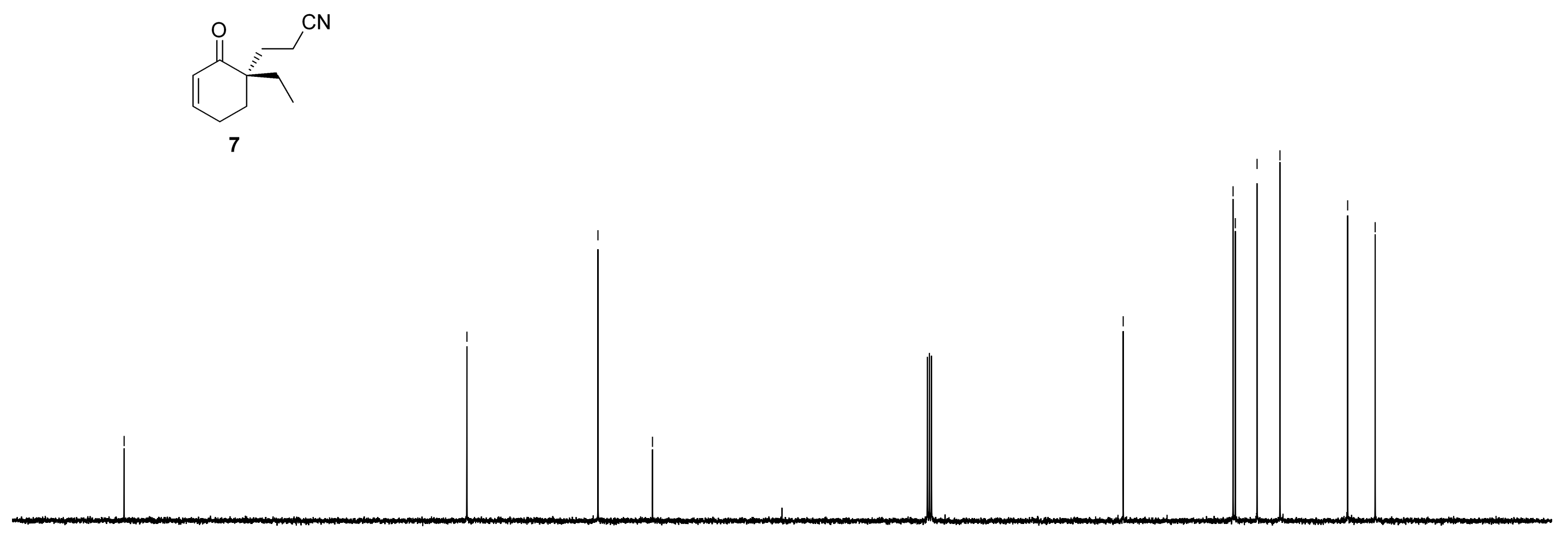

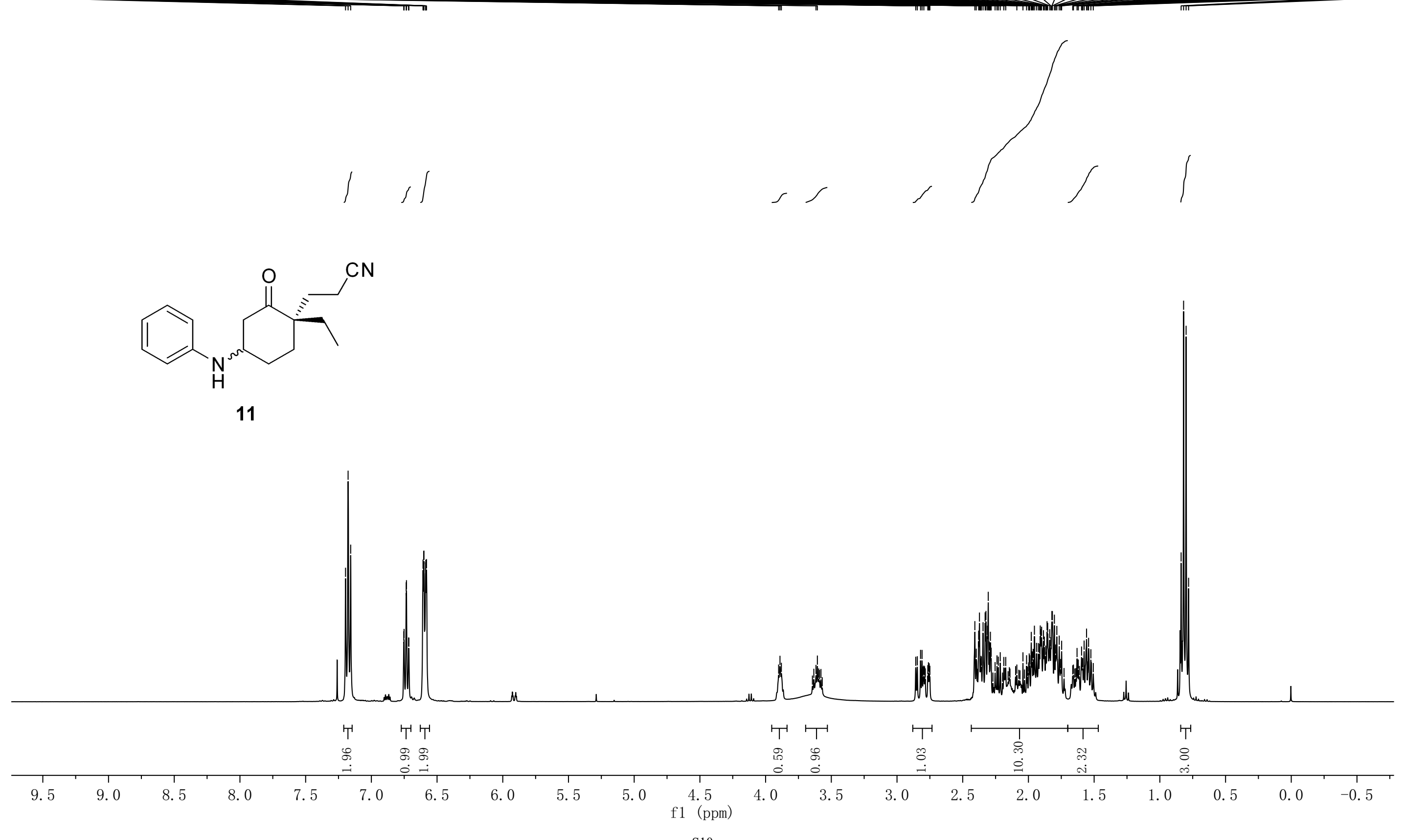


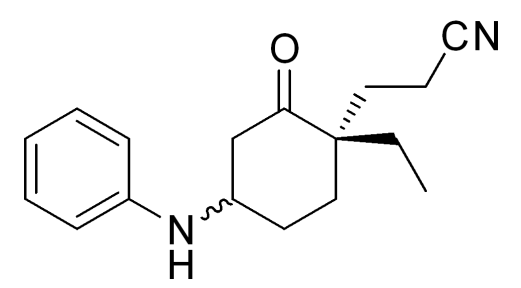

11

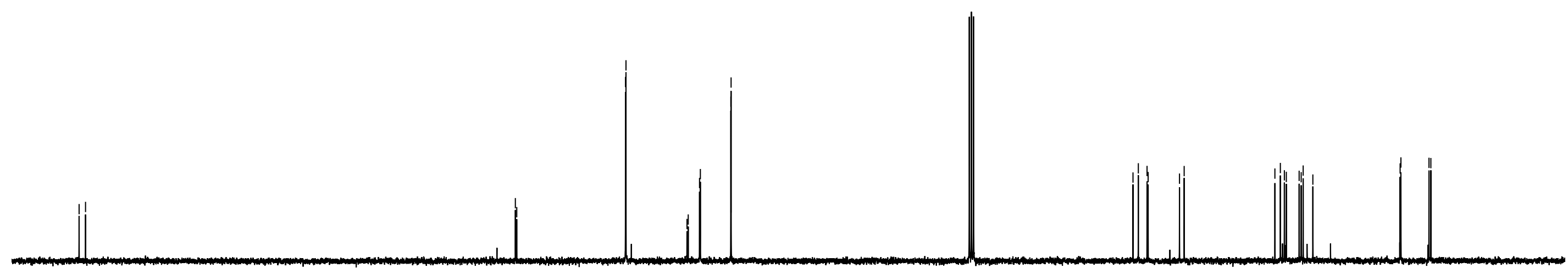

$$
220
$$


ำ

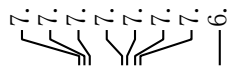

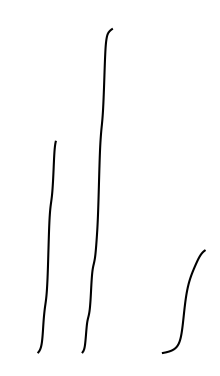

$\overbrace{6}^{C N}$

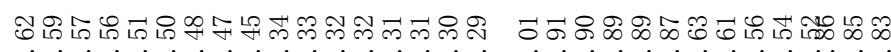

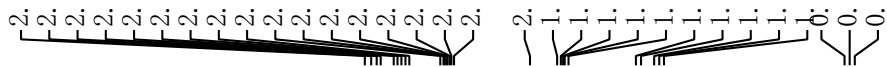

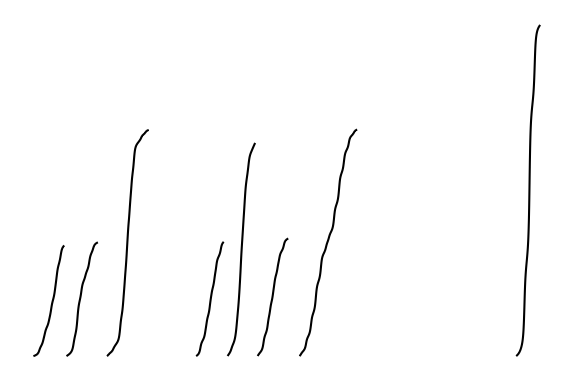

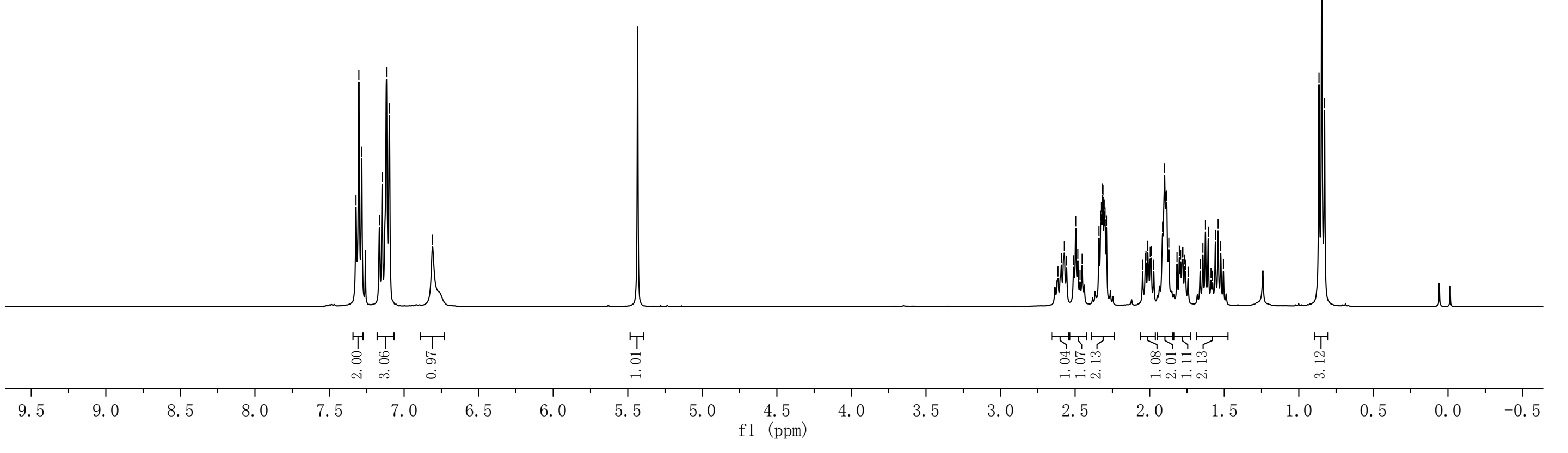



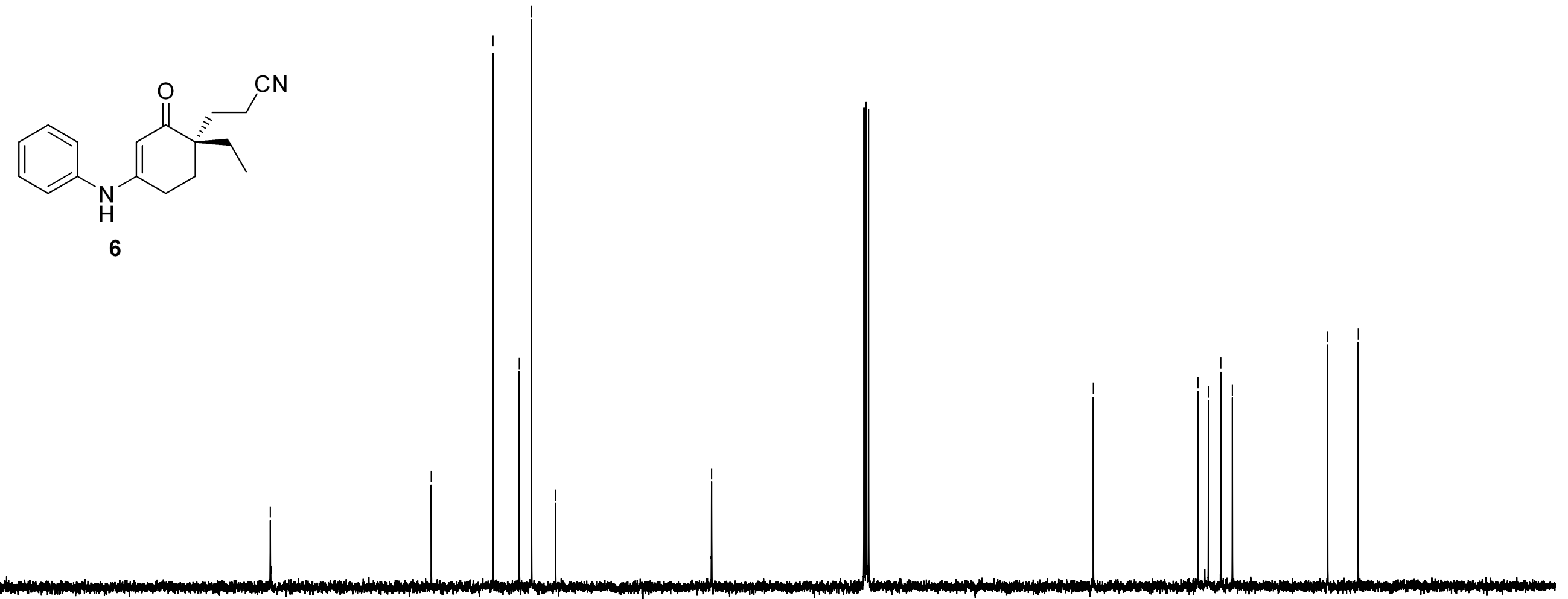

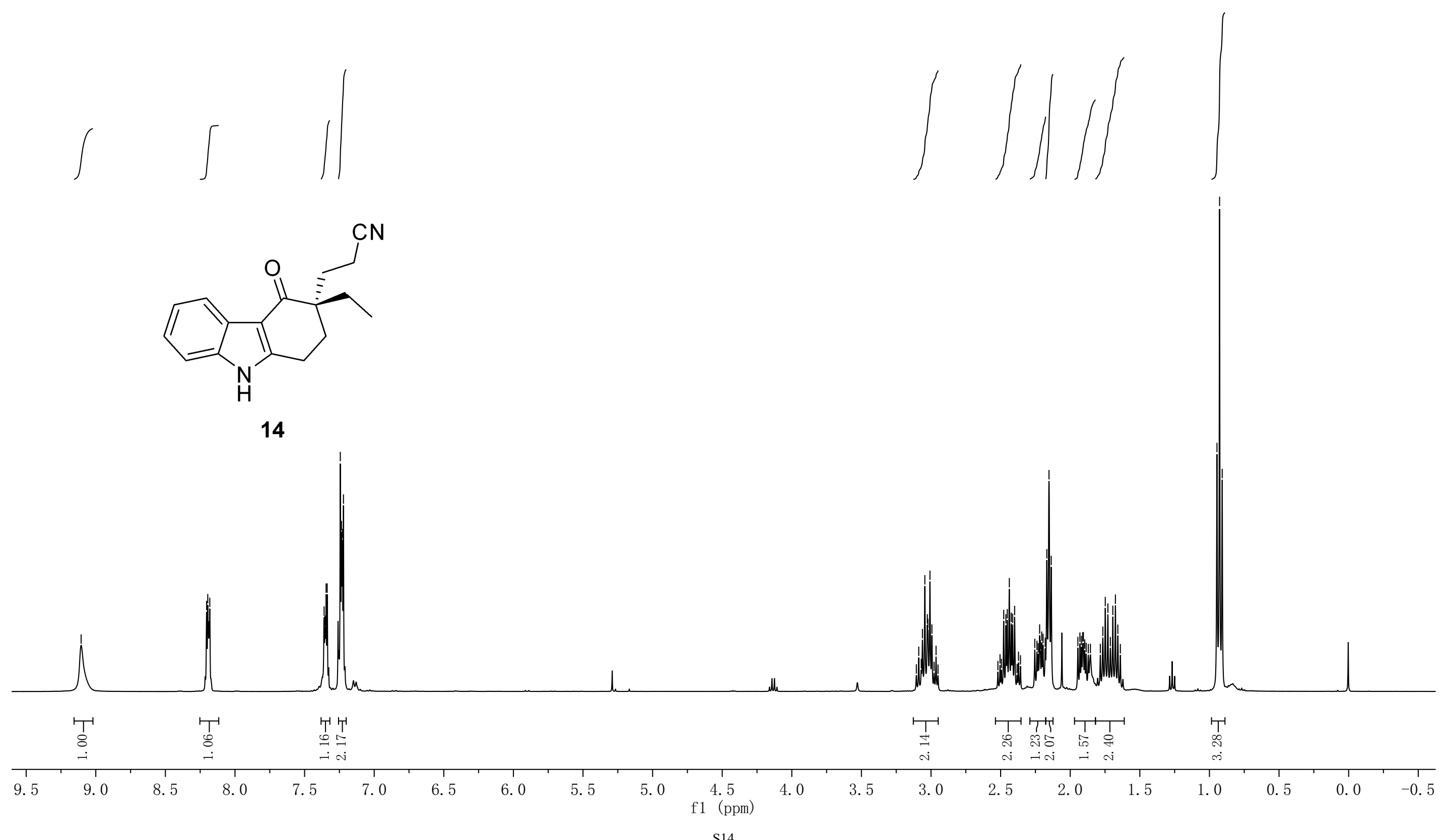

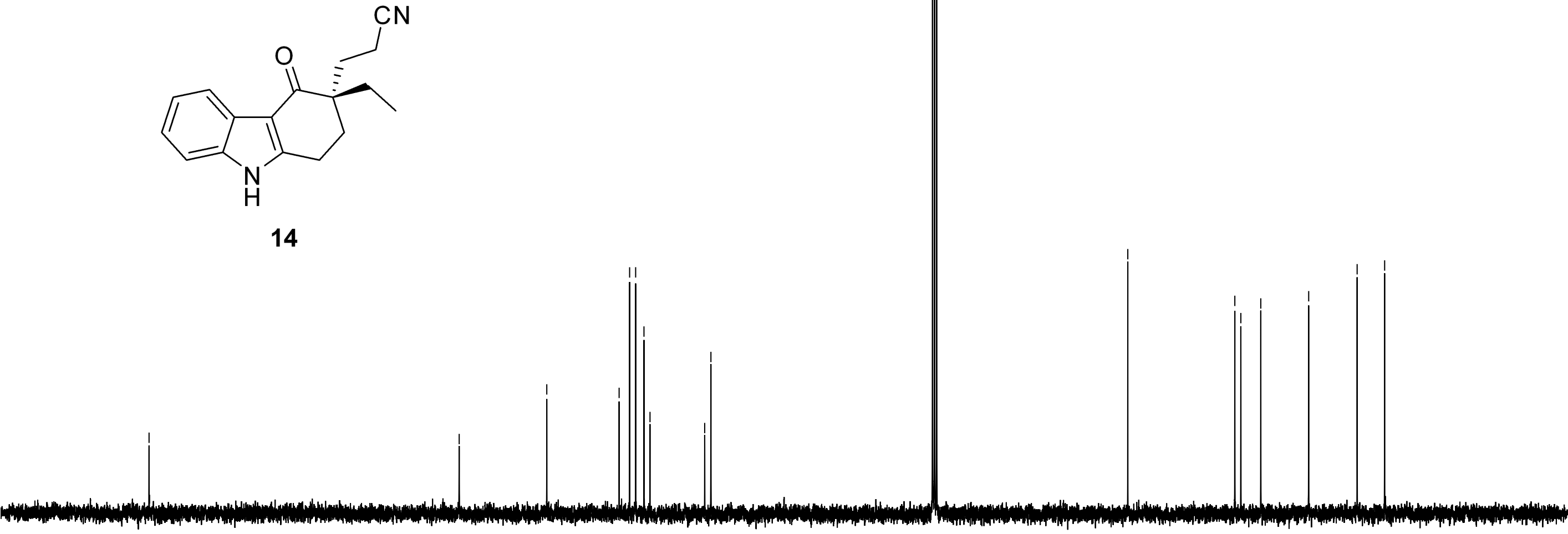
กั่

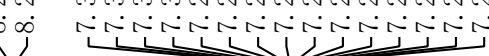

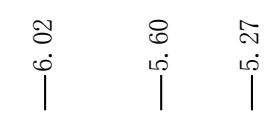

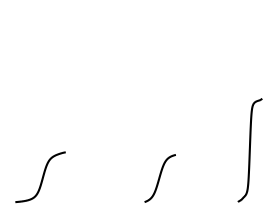<smiles>C1CCCC1</smiles>

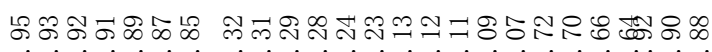

نู
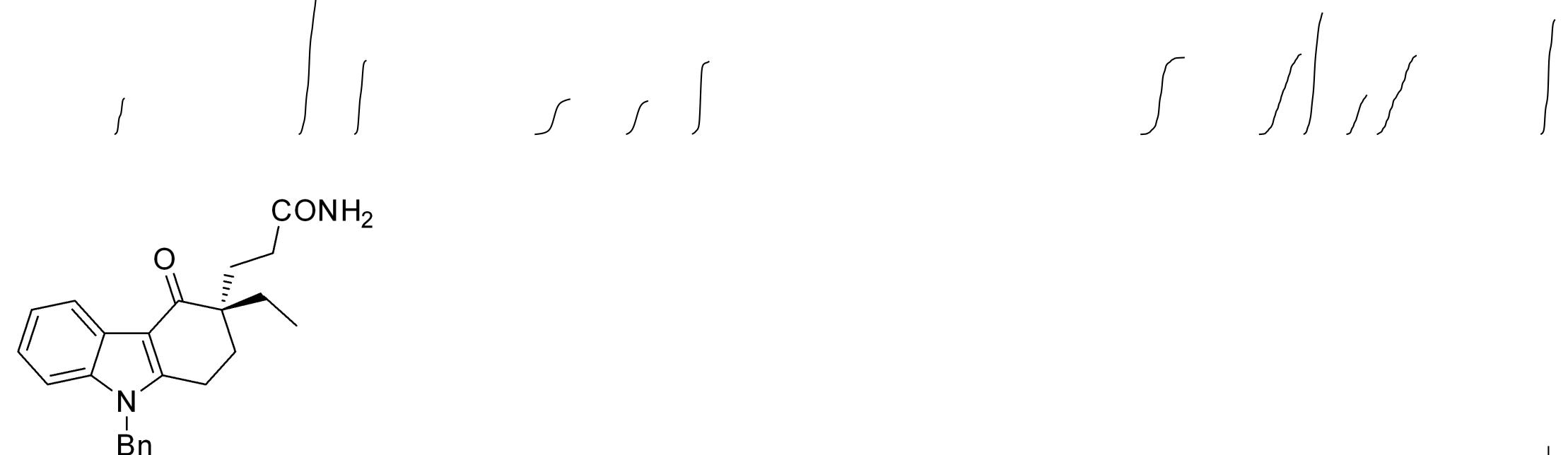

5

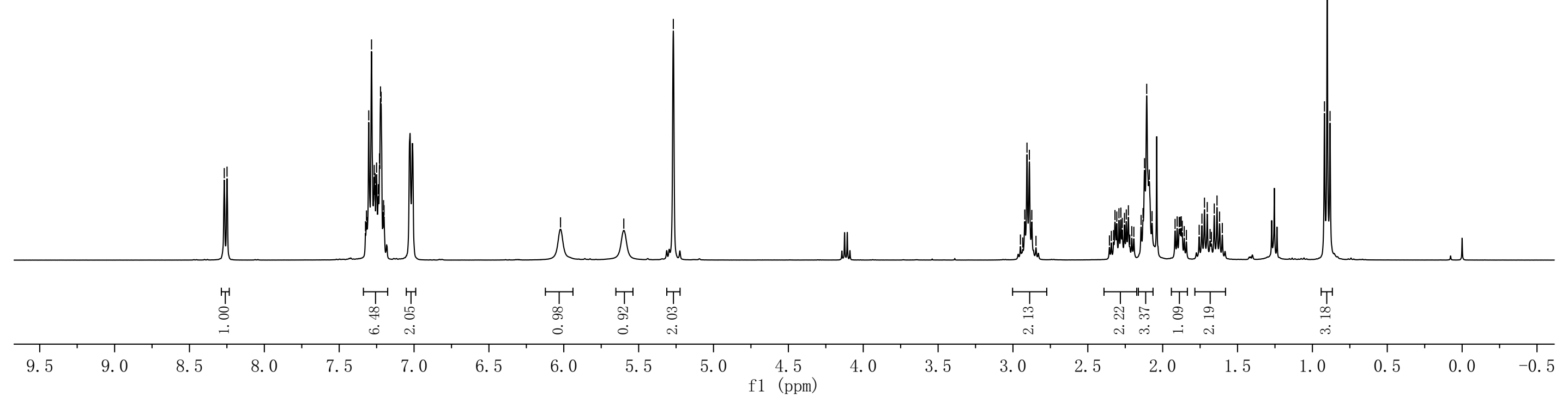




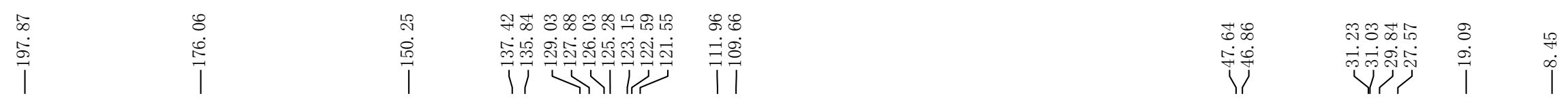
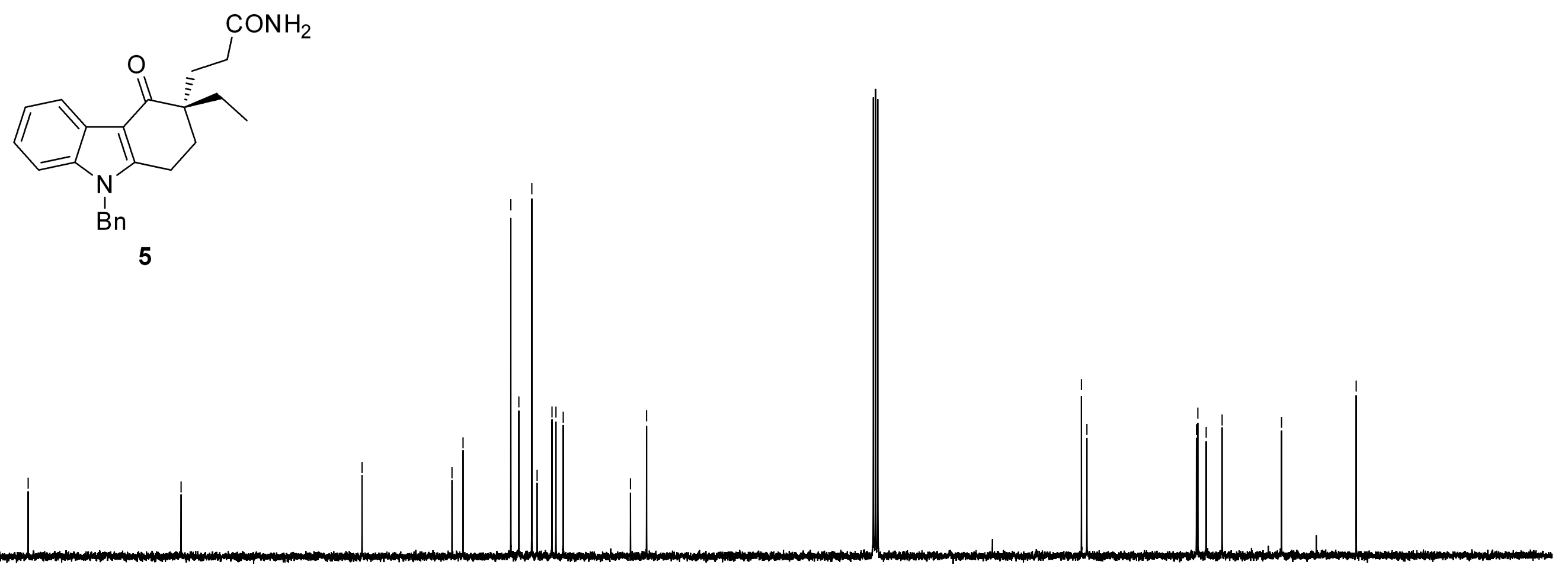

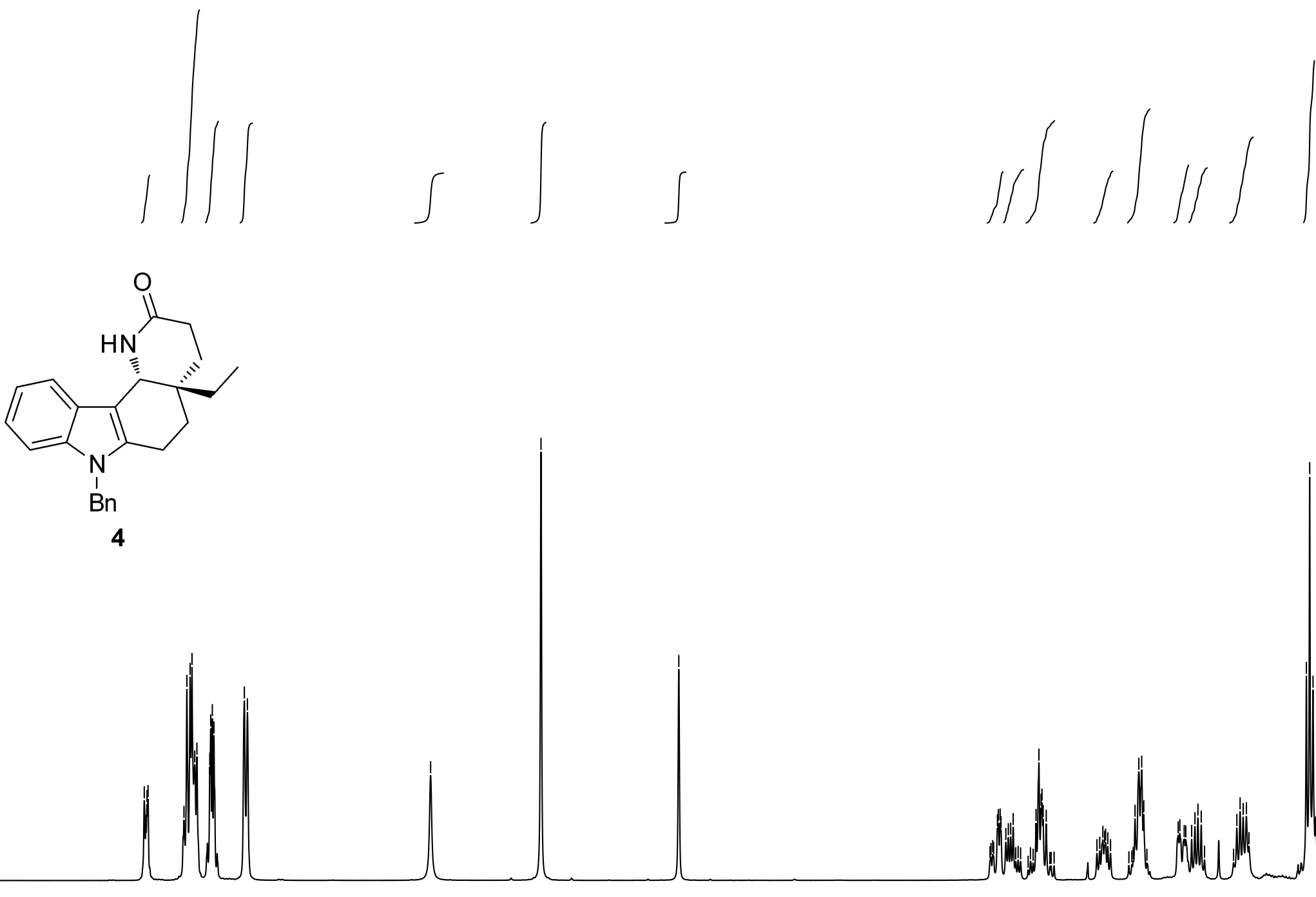

(a) N W

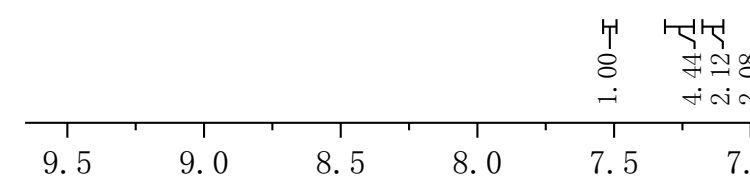

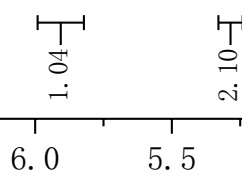

5.0

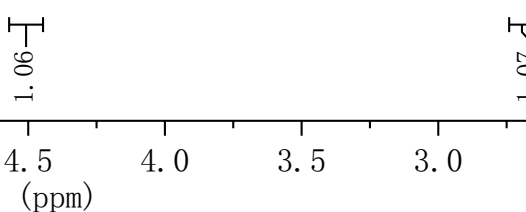

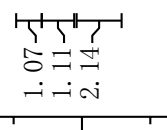

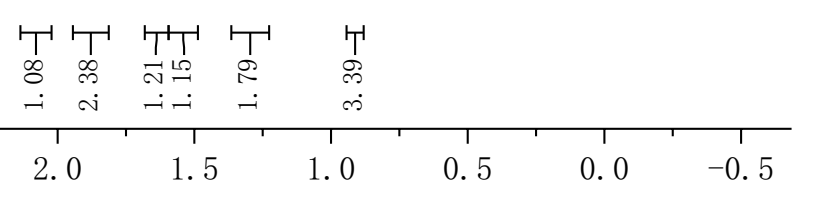



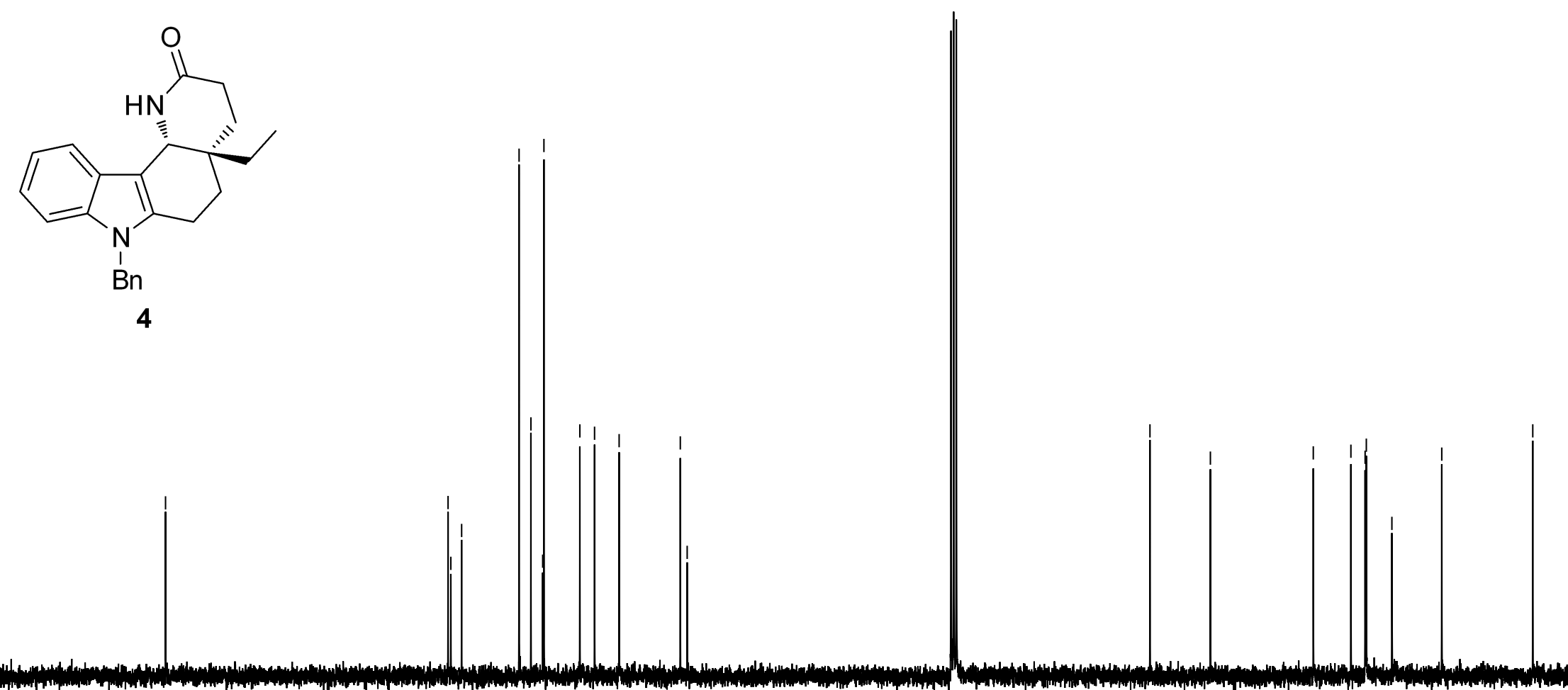

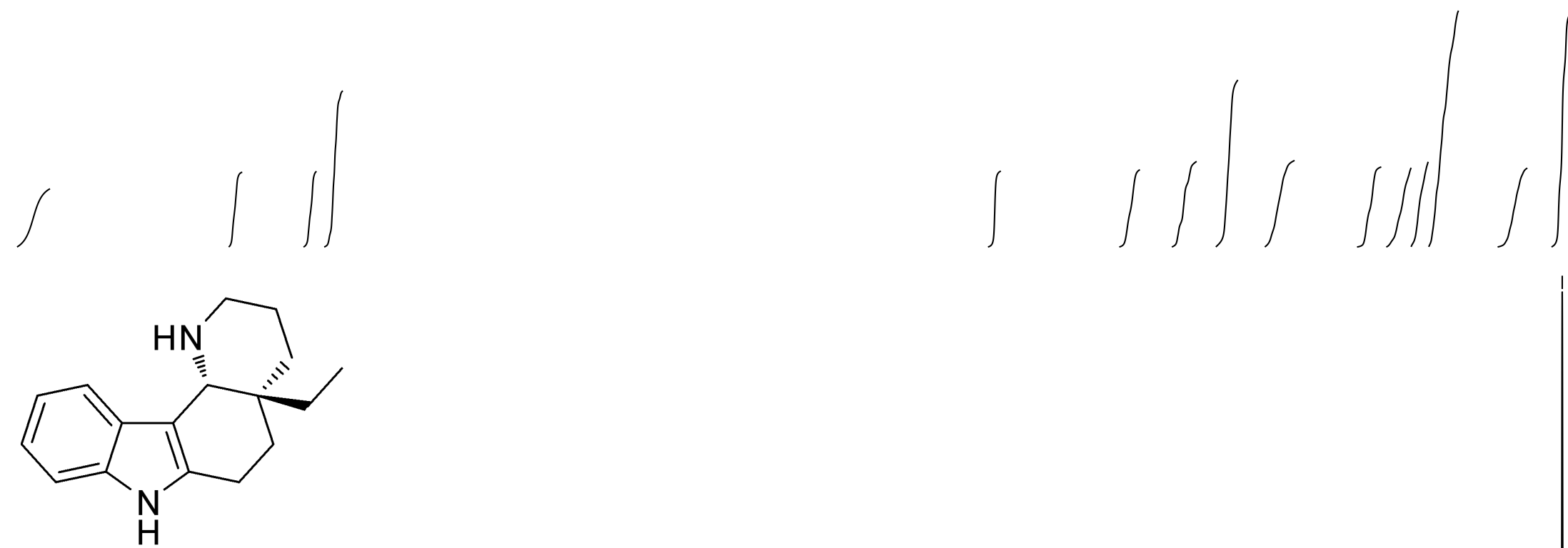

15

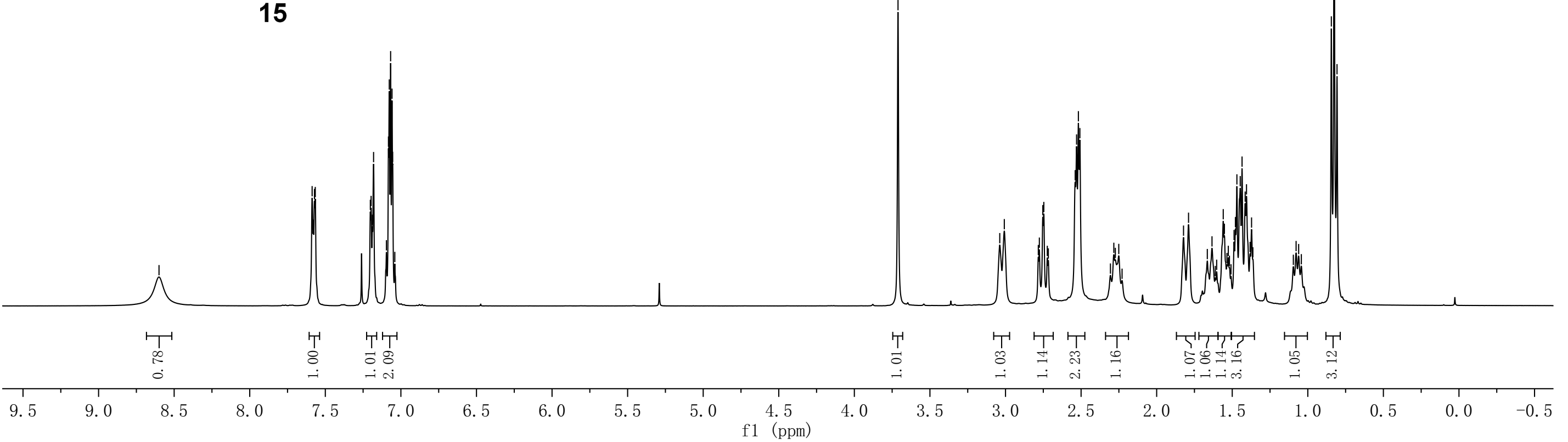




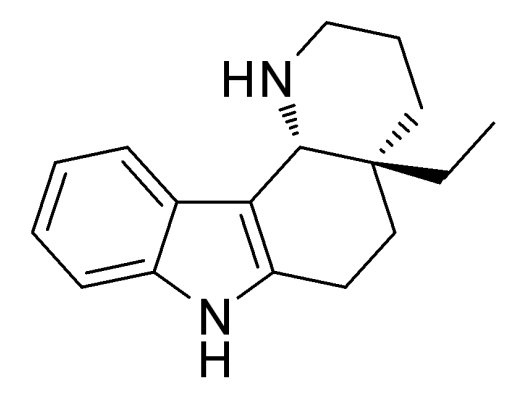

15 

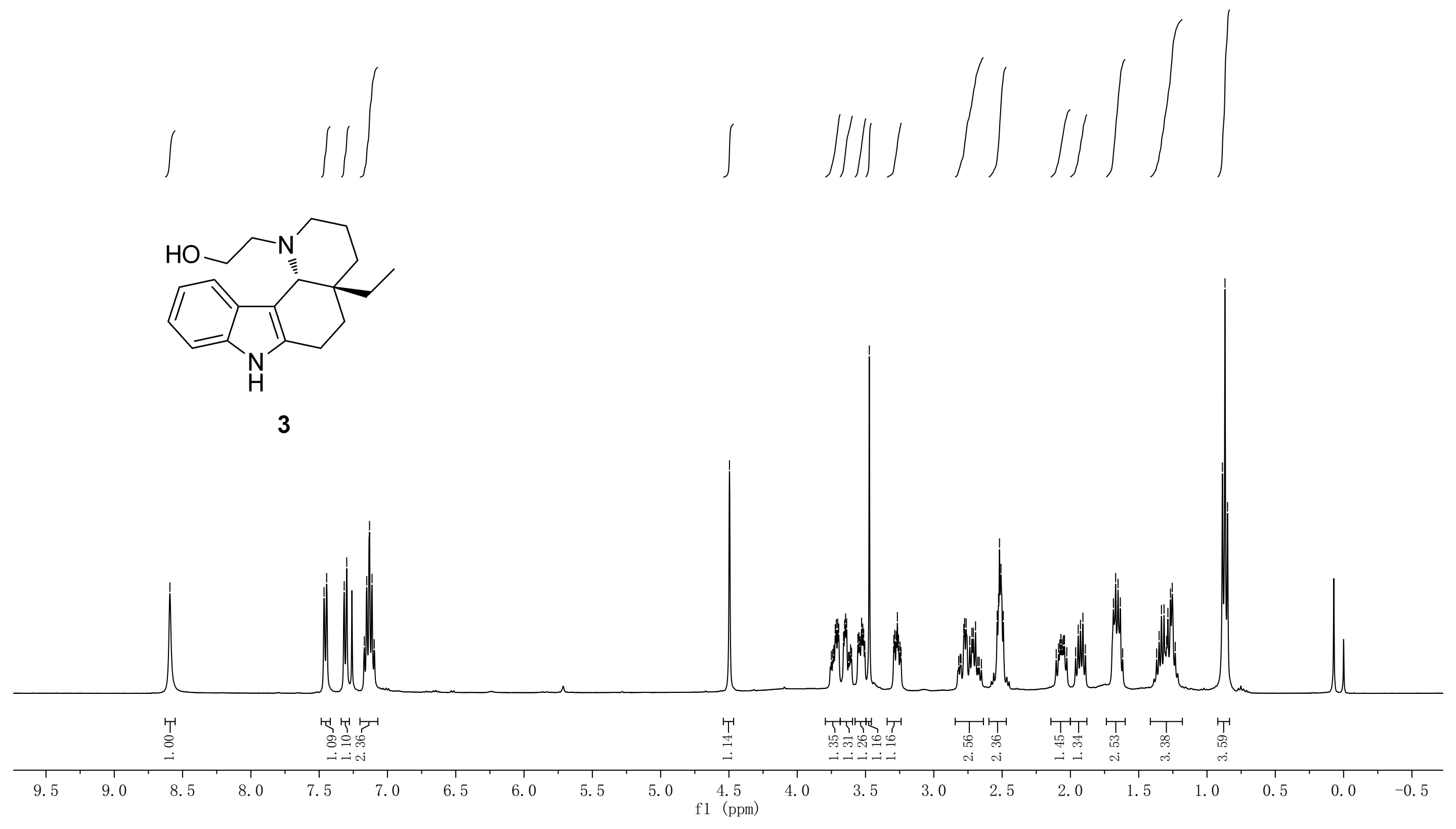

量

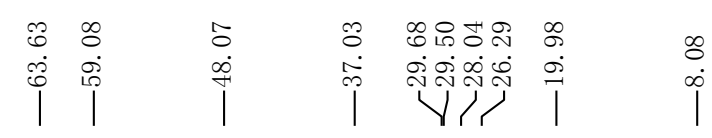
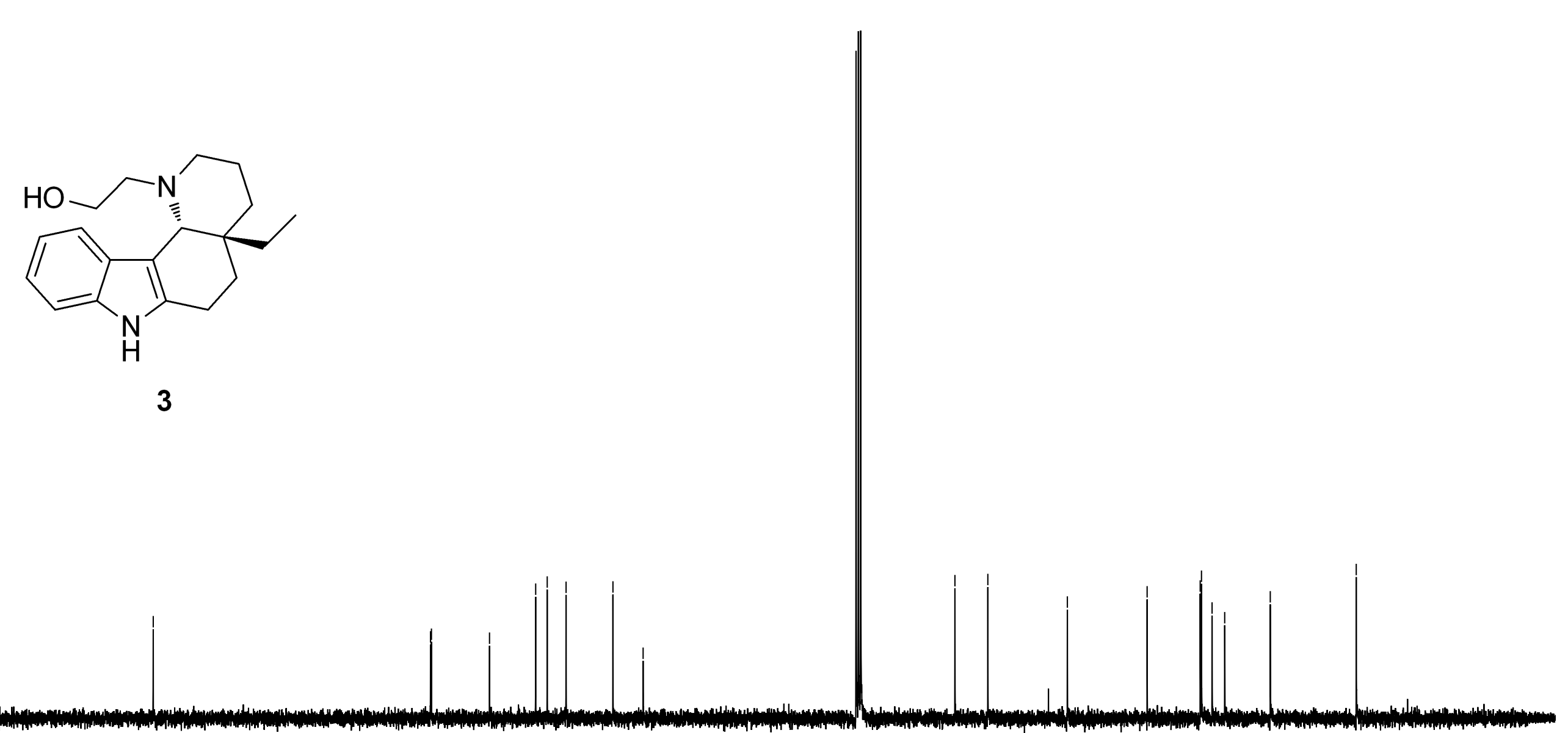

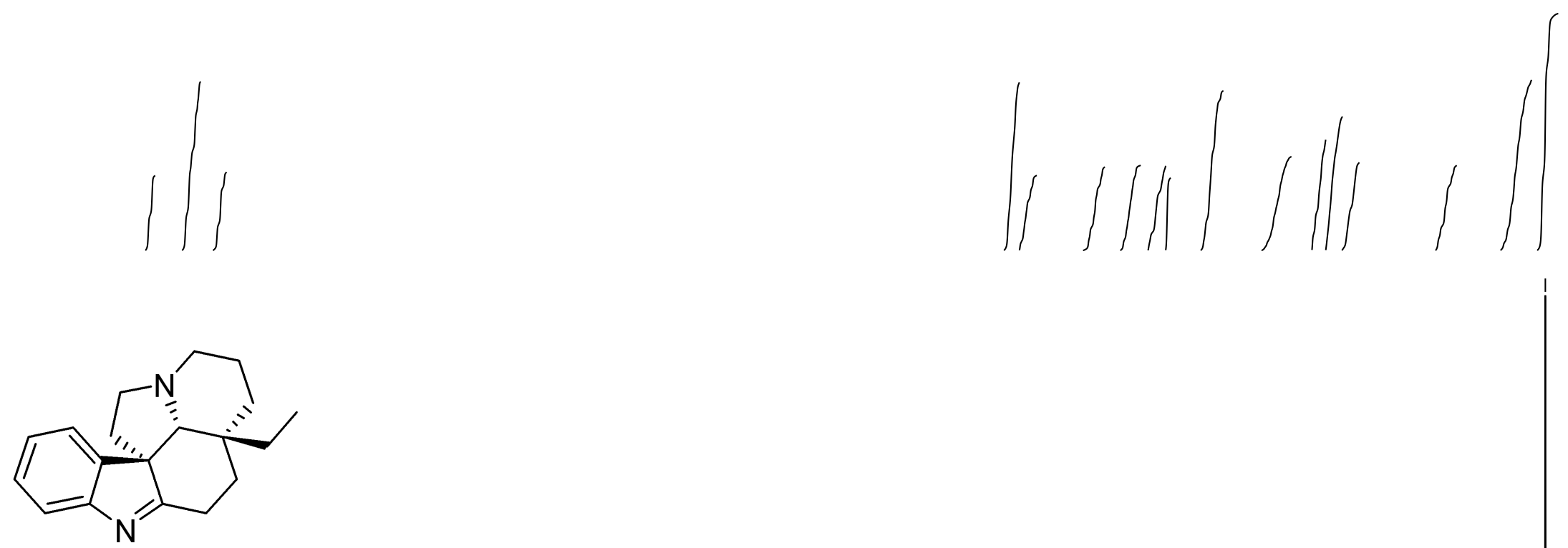

(+)-1,2-dehydroaspidospermidine (2)

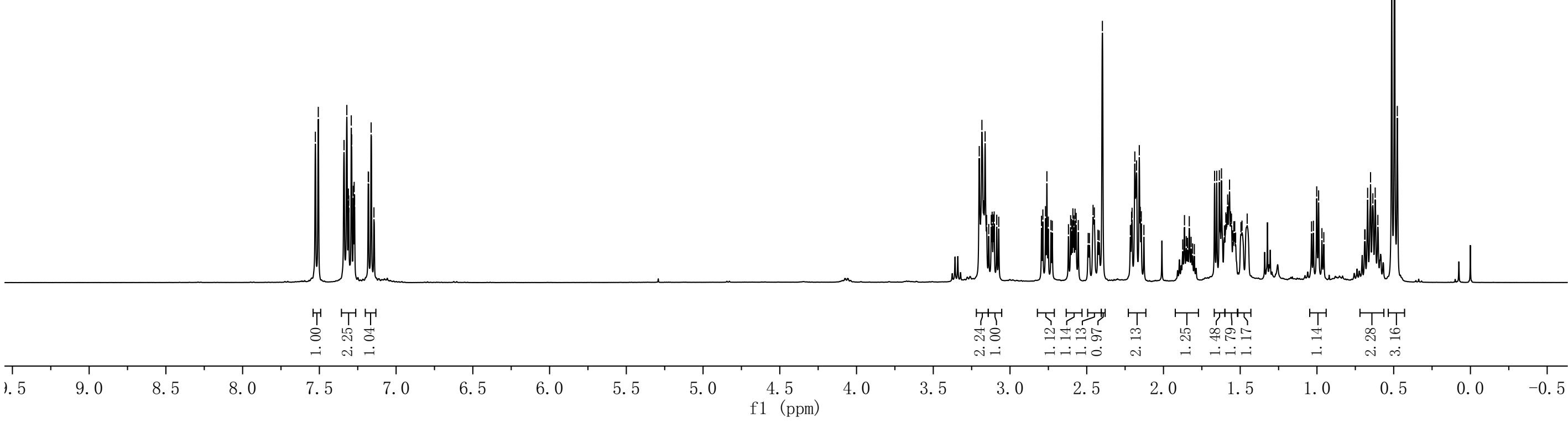




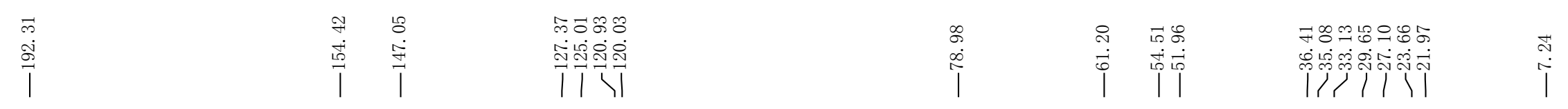

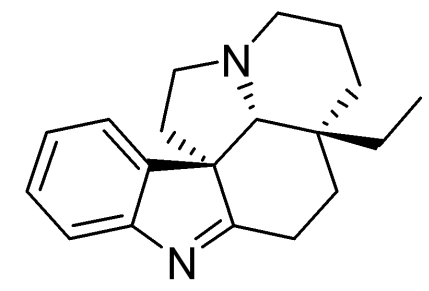

(+)-1,2-dehydroaspidospermidine (2) 


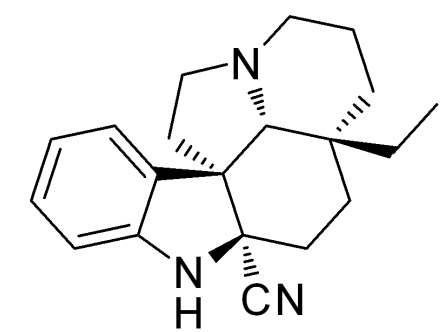

(+)-Winchinine B (1b)

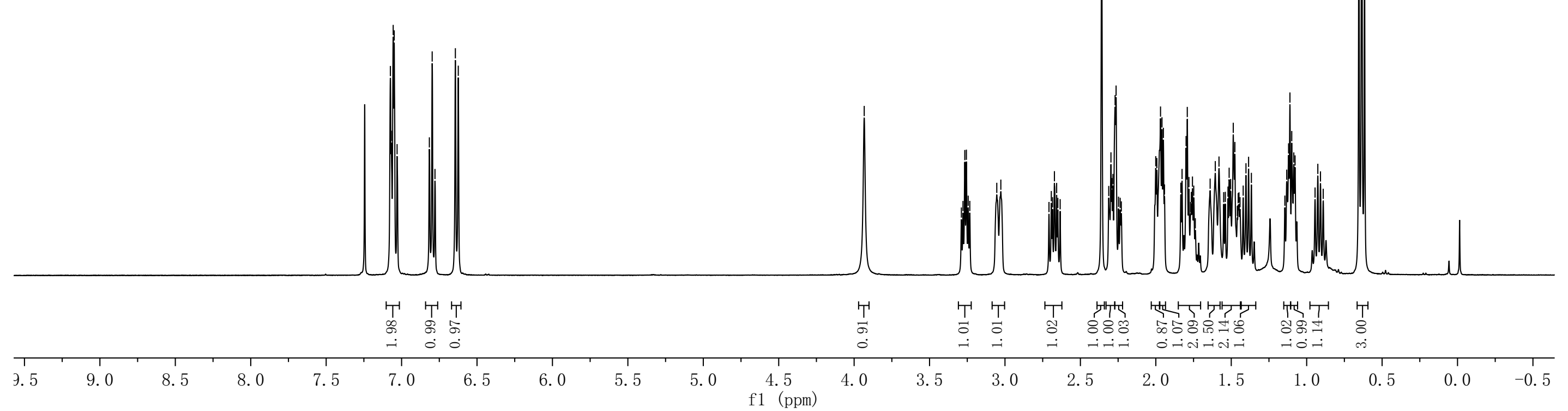




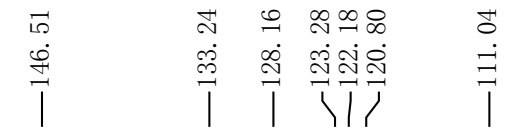

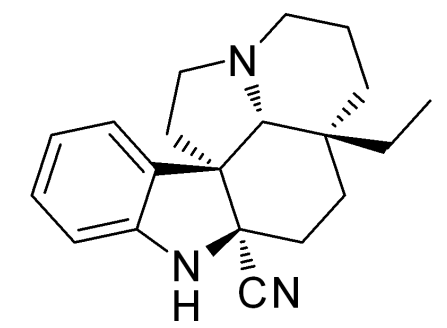

(+)-Winchinine B (1b)

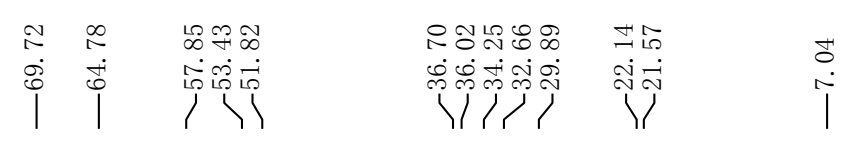

.

$$
\text { T10 }
$$


3. HPLC spectra of S-1

\begin{tabular}{ll}
\hline Column & CHIRALPAK $®$ AY-H \\
\hline Column size & $0.46 \mathrm{~cm} \mathrm{I.D.} \times 25 \mathrm{~cm} \mathrm{~L} \times 5 \mathrm{um}$ \\
Mobile phase & n-Hexane/EtOH=90/10 $(\mathrm{v} / \mathrm{v})$ \\
Injection & $5 \mathrm{uL}$ \\
Flow rate & $1 \mathrm{~mL} / \mathrm{min}$ \\
Wave length & PDA $216 \mathrm{~nm}$ \\
Sample solution & $1 \mathrm{mg} / \mathrm{mL}$ in EtOH \\
\hline
\end{tabular}

\section{Racemic sample:}

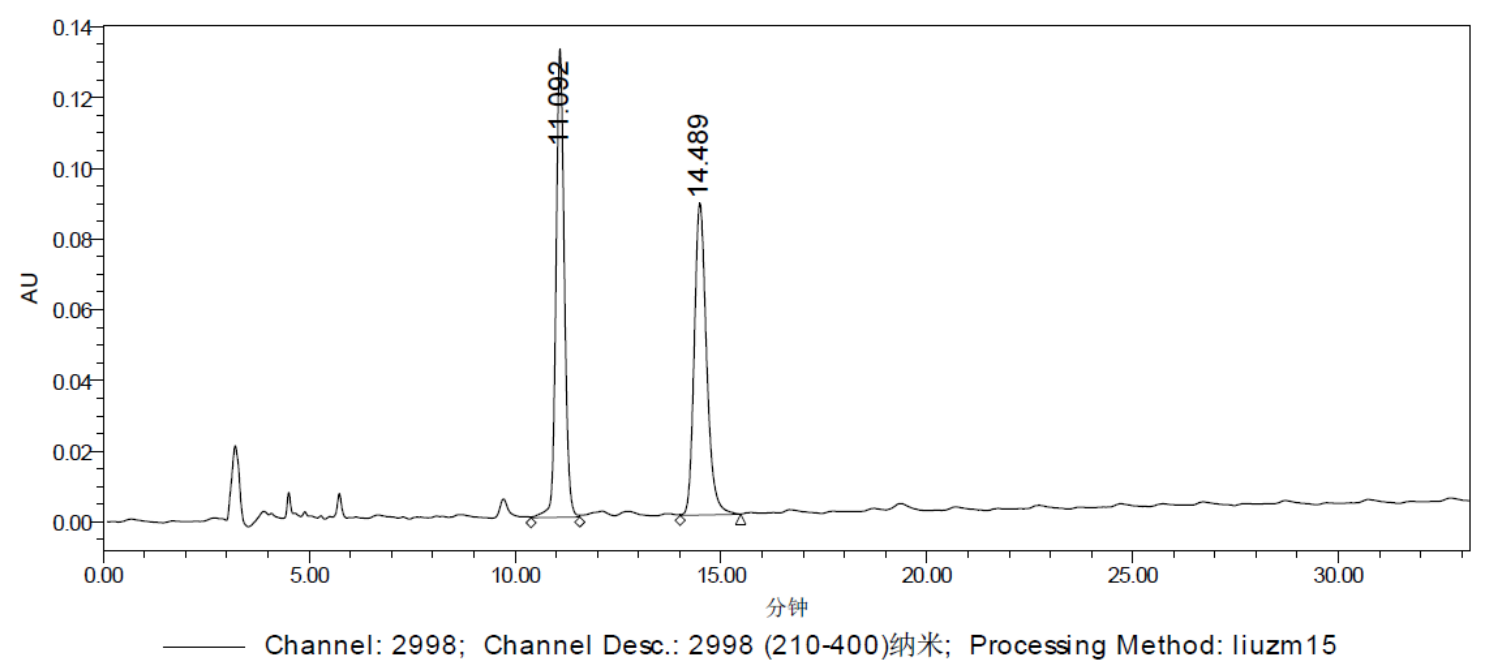

\begin{tabular}{lllll}
\hline Peak No. & Ret. Time $(\mathrm{min})$ & Area & Height & Area \% \\
\hline 1 & 11.092 & 1864118 & 132362 & 50.39 \\
2 & 14.489 & 1835007 & 88168 & 49.61 \\
Total & & 3699125 & 220530 & 100 \\
\hline
\end{tabular}




\section{Chiral sample:}

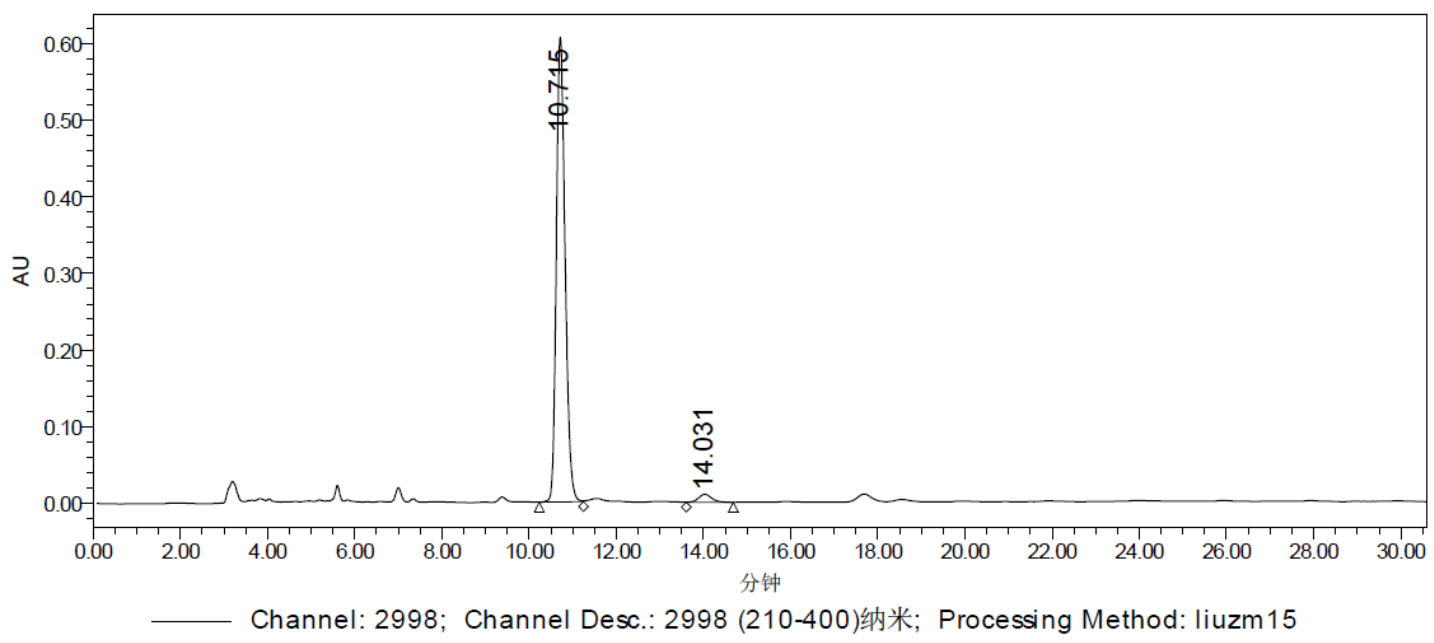

\begin{tabular}{lllll}
\hline Peak No. & Ret. Time $(\mathrm{min})$ & Area & Height & Area \% \\
\hline 1 & 10.715 & 8426718 & 606272 & 97.46 \\
2 & 14.031 & 219480 & 10495 & 2.54 \\
Total & & 8646198 & 616767 & 100 \\
\hline
\end{tabular}

